Planet. Space Sci. 1971, Vol. 19, gp. 1019 to 1023. Pergamon Press. Printed in Northern Iretand

\title{
FURTHER REMARKS ON THE DOUBLE SCATTERING OF THE PLASMA STREAM IN A BI-THERMAL IONOSPHERE
}

(Received in final form 7 December 1970)

Al'pert, Bud'ko and Vas'kov's comments (1970) on our earlier paper (Liu and Hung, 1968) gives us a chance to re-evaluate our earlier analysis. We concur with some of their comments and disagree with the others. In view of the importance of the theory of the plasma far wake, we would like to present a new analysis following essentially the same procedure of integration we used earlier which is different from the approximate method of integration mentioned by Alpert, Bub'ko and Vas'kov (1970).

When the external magnetic field is absent, the perturbation to the ion density in the far wake of a single spherical body is given by (Liu and Hung, 1968):

$$
n^{(1)}(r, \theta, A, \alpha)=-\frac{\pi R_{1}^{2} A^{2} n_{0}}{(2 \pi)^{2} r^{2}} F^{(1)}(\theta, A, \alpha)
$$

where

$$
F^{(1)}(\theta, A, \alpha)=\cos \theta \int_{-1}^{-1} \frac{Z(A t)}{1+\alpha+i \alpha A t Z(A t)} \times \frac{t \mathrm{~d} t}{\left(t^{2}-\sin ^{2} \theta\right)^{3 / 2}}
$$

and

$$
Z(A t)=\frac{1}{\sqrt{\pi i}} \int_{-\infty}^{\infty} \frac{\mathrm{e}^{-u^{2}}}{u-A t} \mathrm{~d} u .
$$

Notice that the branch cut of the integral (2) may be identified as the ine segment $A B$ of the real axis as shown in Fig. 1 where points $A$ and $B$ are prescribed by $(-\sin \theta, 0)$ and $(\sin \theta, 0)$ respectively. For convenience of comparison with the results of integration referred by Al'pert, Bud'ko and Vas'kov (1970), we re-define the angle $\theta$ to comply with their convention. In view of the presence of the branch cut, the ranges of $\theta_{1}$ (at $A$ ) and $\theta_{2}$ (at $B$ ) are precribed as follows: $2 \pi<\theta_{1}<0 ;-\pi<\theta_{2}<\pi$. In the evaluation

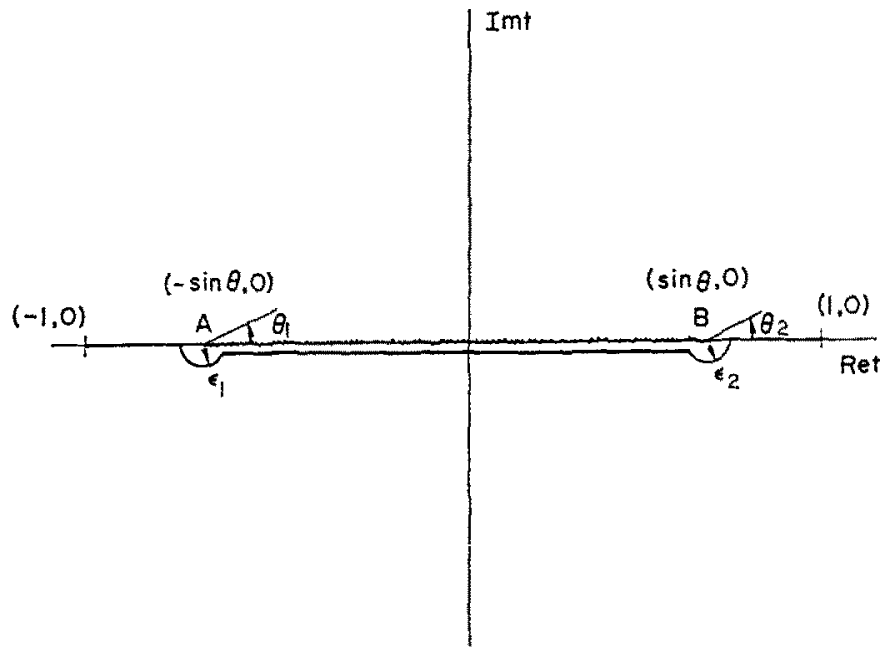

FIG. 1. BRANCH CUT OF THE INTEGRAND (2), CONTOUR OF INTEGRATION (2). 
of the integral (2), the contour of integration as that shown in Fig. 1 and the integral (2) can thus be segmented as follows:

$$
F^{(1)}(\theta, A, x)=\left(1-a^{2}\right)_{\varepsilon_{1}, \varepsilon_{2} \rightarrow 0}^{1 / 2 \lim }\left[\int_{-1}^{-a-\varepsilon_{1}}+\int_{-a-\varepsilon_{1}}^{-a+\varepsilon_{1}}+\int_{-a+\varepsilon_{1}}^{a-\varepsilon_{3}}+\int_{a-\varepsilon_{3}}^{a+\varepsilon_{2}}+\int_{a+\varepsilon_{2}}^{1}\right] \frac{\Phi(A t) t \mathrm{~d} t}{\left(t^{2}-a^{2}\right)^{3 / 2}}
$$

where $a=\sin \theta$ and

$$
\Phi(A t)=\frac{Z(A t)}{1+x+i x A t Z(A t)} .
$$

The integrals in (4) are evaluated by means of integration by parts as follows:

$$
\begin{aligned}
& \operatorname{Lim}_{s_{1} \rightarrow 0} \int_{-1}^{-a-e_{1}} \frac{\Phi(A t) t \mathrm{~d} t}{\left(t^{2}-a^{2}\right)^{3 / 2}}= \operatorname{Lim}_{\varepsilon_{1} \rightarrow 0}\left[\frac{\Phi(-A a)}{\left(2 a \varepsilon_{1}\right)^{1 / 2}}\right]-\frac{\Phi(-A)}{\left(1-a^{2}\right)^{1 / 2}}-\Phi^{\prime}(-A a) \log a \\
&+\Phi^{\prime}(-A) \log \left(1+\sqrt{\left.1-a^{2}\right)}+\int_{1}^{a} \Phi^{\prime \prime}(-A \xi) \log \left(\xi+\sqrt{\left.\xi^{2}-a^{2}\right) \mathrm{d} \xi}\right.\right. \\
& \operatorname{Lim}_{\varepsilon_{1} \rightarrow 0} \int_{-a-\varepsilon_{1}}^{-a+\varepsilon_{1}} \frac{\Phi(A t) t \mathrm{~d} t}{\left(t^{2}-a^{2}\right)^{3 / 2}}=(-1-i) \frac{\Phi(-A a)}{(2 a)^{1 / 2}} \operatorname{Lim}_{s_{1} \rightarrow 0}\left(\frac{1}{\varepsilon_{1}^{1 / 2}}\right)
\end{aligned}
$$

$$
\begin{gathered}
\operatorname{Lim}_{\varepsilon_{1}, \varepsilon_{3} \rightarrow 0} \int_{-a+s_{1}}^{a-\varepsilon_{2}} \frac{\Phi(A t) t \mathrm{~d} t}{\left(t^{2}-a^{2}\right)^{3 / 2}}=\lim _{\varepsilon_{1}, \varepsilon_{2} \rightarrow 0}\left[i \frac{\Phi(-A a)}{\left(2 a \varepsilon_{1}\right)^{1 / 2}}-i \frac{\Phi(A a)}{\left(2 a \varepsilon_{2}\right)^{1 / 2}}\right]+i\left\{\frac { \pi } { 2 } \left[\Phi^{\prime}(A a)\right.\right. \\
\left.\left.-\Phi^{\prime}(-A a)\right]-\int_{0}^{a}\left[\Phi^{\prime \prime}(A \xi)-\Phi^{\prime \prime}(-A \xi)\right] \sin ^{-1} \frac{\xi}{a} \mathrm{~d} \xi\right\} \\
\operatorname{Lim}_{\varepsilon_{3} \rightarrow 0} \int_{a-\varepsilon_{2}}^{a+\varepsilon_{2}} \frac{\Phi(A t) t \mathrm{~d} t}{\left(t^{2}-a^{2}\right)^{3 / 2}}=(-1+i) \frac{\Phi(A a)}{(2 a)^{1 / 2}} \operatorname{Lim}_{\varepsilon_{2} \rightarrow 0}\left(\frac{1}{\varepsilon_{3}^{1 / 2}}\right)
\end{gathered}
$$

$$
\begin{aligned}
\operatorname{Lim}_{\varepsilon_{2} \rightarrow 0} \int_{a+\varepsilon_{2}}^{1} \frac{\Phi(A t) t \mathrm{~d} t}{\left(t^{2}-a^{2}\right)^{3 / 2}}=\operatorname{Lim}_{\varepsilon_{3} \rightarrow 0}\left[\frac{\Phi(A a)}{\left(2 a \varepsilon_{2}\right)^{1 / 2}}\right] & -\frac{\Phi(A)}{\left(1-a^{2}\right)^{1 / 2}}+\Phi^{\prime}(A) \log \left(1+\sqrt{1-a^{2}}\right) \\
& -\Phi^{\prime}(A a) \log a-\int_{a}^{1} \Phi^{\prime \prime}(A \xi) \log \left(\xi+\sqrt{\xi^{2}-a^{2}}\right) \mathrm{d} \xi
\end{aligned}
$$

(b)

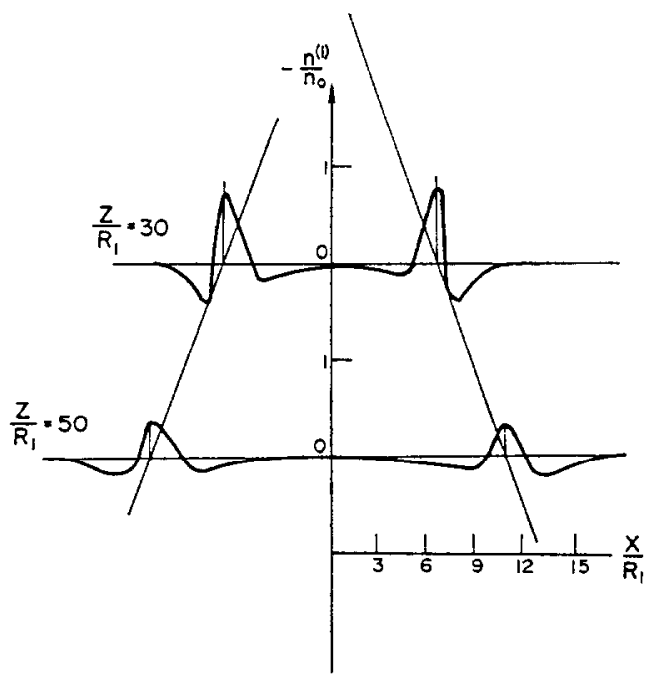

(a)

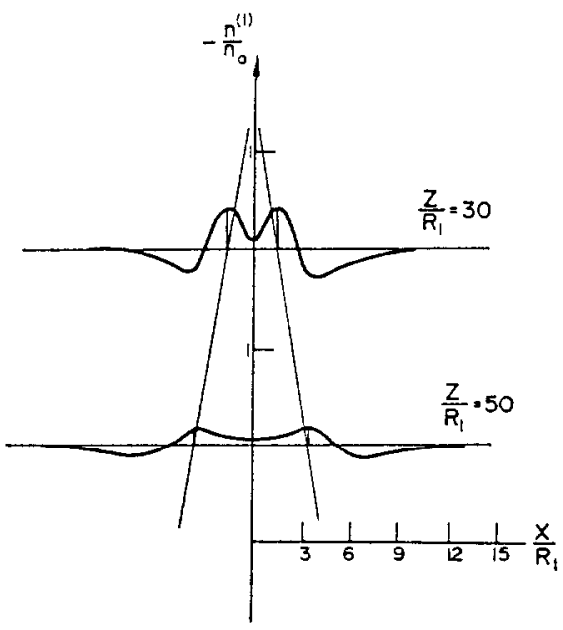

FIG. 2. ION DENSITY PERTURBATIONS IN THE FAR WAKE BEHIND A SPHERICAL BODY.

(a) Speed ratio, $A=8$; Temperature ratio, $\alpha=1$. (b) Speed ratio, $A=8$; Temperature ratio, $\alpha=10$. 
Note that $\operatorname{Re} \Phi(A t)$ is an even function of $t$ and $\operatorname{Im} \Phi(A t)$ is an odd function, where $\Phi(A t)=\operatorname{Re} \Phi(A t)+$ $\operatorname{iIm} \Phi(A t)$. Substituting (6)-(10) into (4) and also making use of the characteristics of $\Phi(A t)$, we have

$$
\begin{aligned}
& F^{(1)}(\theta, A, \alpha)=2 \cos \theta\left\{-\frac{\operatorname{Re} \Phi(A)}{\left(1-\sin ^{2} \theta\right)^{1 / 2}}-\operatorname{Re} \Phi^{\prime}(A \sin \theta) \log \sin \theta\right. \\
& +\operatorname{Re} \Phi^{\prime}(A) \log \left(1+\sqrt{1-\sin ^{2} \theta}\right)-\int_{\sin \theta}^{1} \operatorname{Re} \Phi^{\prime \prime}(A \xi) \log \left(\xi+\sqrt{\xi^{2}-\sin ^{2} \theta}\right) d \xi \\
& \left.\quad-\frac{\pi}{2} \operatorname{Im} \Phi^{\prime}(A \sin \theta)+\int_{0}^{\sin \theta} \operatorname{Re} \Phi^{\prime \prime}(A \xi) \sin ^{-1}\left(\frac{\xi}{\sin \theta}\right) \mathrm{d} \xi\right\} .
\end{aligned}
$$

The integrals of (11) are evaluated numerically by means of digital computer (IBM 360). The results of the ion density perturbation $\left(-n^{(1)} / n_{0}\right)$ for two different ionospheres $\left[\alpha\left(\equiv T_{e} / T_{i}\right)=1\right.$ and $\left.\alpha=10\right]$ with speed ratio $A$ ( $\equiv$ body velocity/ion thermal velocity) $=8$ are shown in Fig. 2.*

In our previous calculation (Liu and Hung, 1968), the ranges of values for $\theta_{1}$ and $\theta_{2}$ at $A$ and $B$ respectively were not appropriately prescribed; there were a!gebraic errors incurred in the evaluation of $R e$ and $I m$ parts of the integrand of (5) and also there was an oversight of a factor $(2 \pi)^{-2}$ in the figures (Liu and Hung, 1968).

It is noted from Fig. 2 that the two rarefaction pips $\left(-n^{(1)} / n_{0}\right)$ are so located in the wake that at far downstream it appears that a conical surface of maximum rarefaction disturbances can be defined where the conical angle is approximately given by $\sin ^{-1}\left(C_{s} / V\right)$ where $V$ denotes the body velocity; $C_{s}$, the ion acoustic velocity which equals $\left(K T_{e} / m_{i}\right)^{1 / 2}$. It appears that the far wake has a wavelike character, in the sense that the disturbances propagate as a result of collective interaction of all the plasma particles through which they pass rather than as a solitary wave which moves into an undisturbed ambient plasma. It is apparent that there is a condensation perturbation associated with the rarefaction perturbation. These results appear to agree with the experimental results available to us (see Fig. 11 (non-isothermal), and Fig. 16 (isothermal), Hester and Sonin, 1970). This is, however, contrary to the conclusion of Al'pert, Bud'ko and Vas'kov (1970), who claim the absence of condensation of particles outside the rarefaction region.

The ion distribution in the far wake behind the second body can be obtained as follows:

$$
(\mathbf{u}-\mathbf{v}) \cdot \frac{\partial}{\partial \mathbf{r}}\left(f^{(1)}+f^{(2)}\right)-\frac{e}{m_{i}} \frac{\partial\left(\varphi_{1}+\varphi_{2}\right)}{\partial \mathbf{r}} \cdot \frac{\partial f_{01}}{\partial \mathbf{u}}=-\frac{\mathbf{r} \cdot \mathbf{v}}{r} f_{01} \delta\left(\mathbf{r}-R_{2}\right)
$$

where $f_{01} \propto \exp \left[-u^{2} / v_{1}{ }^{2}-e \varphi_{1} / K T_{i}\right]$ is the free stream ion distribution for the second body, and $f^{(2)}$ is the perturbation of ion distribution function deviated from $f_{01}$. Substituting the expression of $f_{01}$ into the Vlasov equation and dropping terms of the order $O\left(\delta^{2}\right)$ or higher, we have

$$
(\mathbf{u}-\mathbf{v}) \cdot \frac{\partial}{\partial \mathbf{r}}\left(f^{(1)}+f^{(2)}\right)+\frac{e}{K T_{i}} f_{0} \mathbf{u} \cdot \frac{\partial\left(\varphi_{1}+\varphi_{2}\right)}{\partial \mathbf{r}}=-\frac{\mathbf{r} \cdot \mathbf{v}}{r} f_{01} \delta\left(r-R_{2}\right),
$$

if the second body is approximated by a source of surface potential $\varphi_{1}$. After the application of Fourier transform to (13) and simple calculations, we obtain

$$
f_{k}^{(1)}+f_{k}^{(2)}+\frac{e}{K T_{i}} f_{0} \frac{\mathrm{k} \cdot \mathrm{v}}{\mathrm{k} \cdot(\mathbf{u}-\mathrm{v})}\left(\varphi_{1 k}+\varphi_{2 k}\right)=\frac{i \pi R_{2}=v f_{0} \exp \left(-\frac{e \varphi_{R_{2}}}{K T_{i}}\right)}{\mathrm{k} \cdot(\mathrm{u}-\mathrm{v})}
$$

where $\varphi_{R_{2}}=$ electrostatic potential at $r=R_{2}$. Making use of Poisson's equation and the condition of quasi-neutral in the far wake, we obtain

$$
\frac{e\left(p_{1 k}+p_{2 k}\right)}{K T_{0}}=\frac{n_{i}^{(2)}}{n_{01 k}}
$$

The Equation (14) integrated over the velocity space leads to

$$
n_{k}^{(1)}+n_{k}^{(2)}+\frac{n_{k}^{(2)} \alpha}{n_{01 k}} \int f_{0} \frac{\mathrm{k} \cdot \mathrm{u}}{\mathrm{k} \cdot(\mathbf{u}-\mathrm{v})} \mathrm{du}=i \pi R_{\mathbf{2}}{ }^{2} v \exp \left(-\frac{e \varphi_{R_{2}}}{K T_{i}}\right) \int \frac{f_{0} \mathrm{du}}{\mathrm{k} \cdot(\mathbf{u}-\mathbf{v})}
$$

* Bud'ko's results (1966) are shown as dotted curve in Fig. 2a (isothermal, $z / R_{1}=30$ ) for comparison. Qualitative agreements between result (Fig. 2b) and Bud'ko's (1970) at different $T_{e} / T_{j}$ are also noted. 
(b)

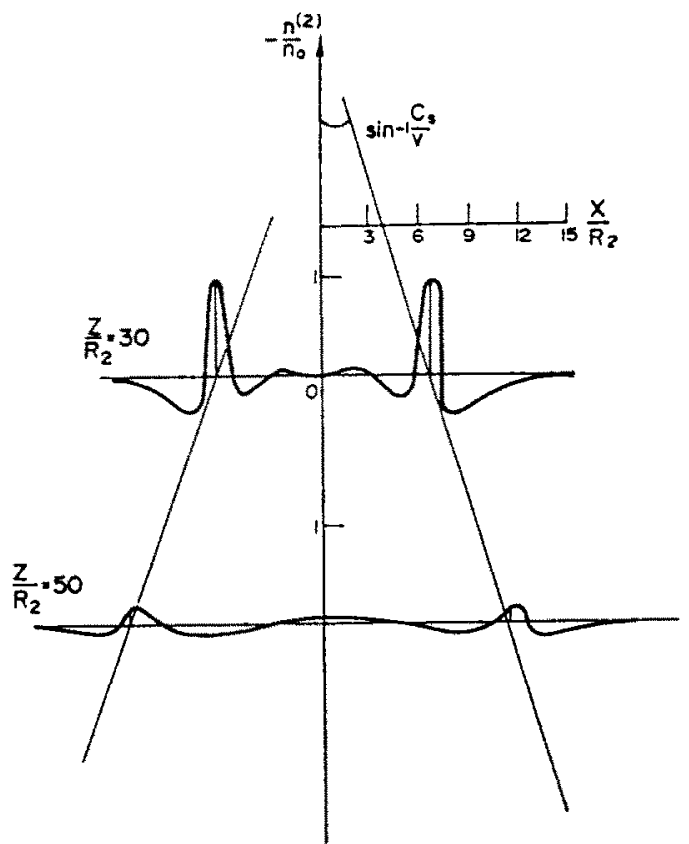

(a)

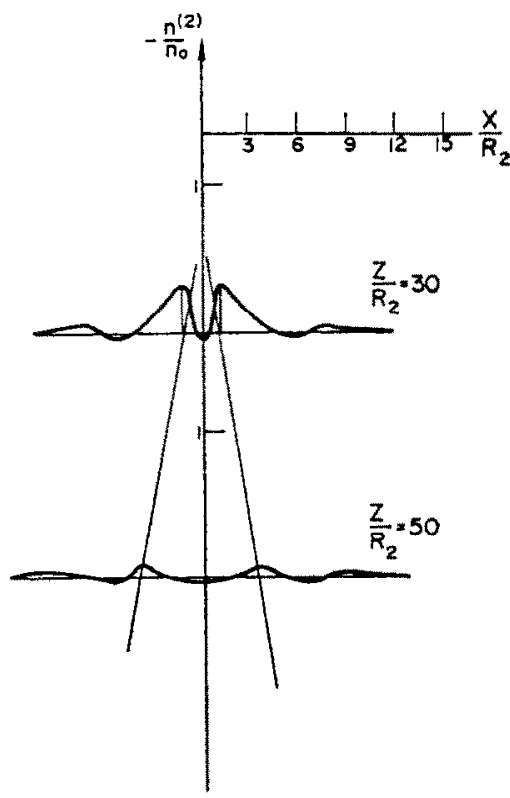

Fig. 3. ION DENSITY PERTURBations IN THE FAR WAKE BEHIND TWO ALIGNED SPHERES. (a) Speed ratio, $A=8$; Temperature ratio, $\alpha=1$. (b) Speed ratio, $A=8$; Temperature ratio, $\alpha=10$.

which can be rearranged and combined with (15) to give

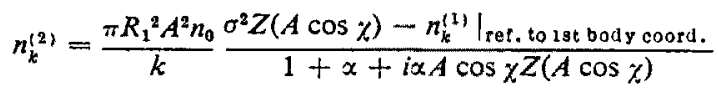

where

$$
\sigma=\frac{R_{1}}{R_{2}} \exp \left(-e \varphi_{E 2} / K T_{i}\right)
$$

and

$$
\left.n_{k}^{(1)}\right|_{16 t \text { body }} \ll \sigma^{2} Z(A \cos z)
$$

The results of ion density perturbation $\left(-n^{(2)} / n_{0}\right)$ in the far wake of the second body are shown in Fig. 3 . It is noted that the same relation between the location of the maximum rarefaction pips and the speed ratio $V / C$, as that of the first body holds. The presence of the first body, with its associated plasma disturbances in the far wake, however, tends to enhance the magnitude of the rarefaction pips behind the second body as shown in Fig. 3.

In the analysis of ion density perturbation with the presence of an external magnetic field aligned with the direction of motion of the sphere, we give results for two simple cases, namely (i) when $\left(k_{\|} V-N \Omega_{i}\right) /$ $k_{\|\|} v_{i} \ll 1$ and (ii) when $\left(k_{\|} V-N \Omega_{i} / k_{\|} v_{i} \gg 1\right.$. Under these conditions the power series expansion of the $Z$ function are justified. Al'pert, Bud'ko and Vas'koy's comment (1970) concerning the singularity of the integrand must originate from misunderstanding of the assumed conditions of our analysis. 


\section{REFERENCES}

AL'PeRT, YA. I., BuD'ko, N. I. and VAs'kov, V. V. (1970). Planet. Space Sci. 18, 1843.

BUd'ko, N. I. (1966). Geomag. Aeron. 6, 787.

BuD'ko, N. I. (1970). Soviet phys. JETP 30, 375.

Hester, S. D. and Sontn, A. A. (1970). AIAA J. 8, 1090.

Liv, V. C. and HUNG, R. J. (1968). Planet. Space Sci. 16, 8+5. 